\title{
Tuberculosis en pacientes con diabetes mellitus: Un estudio epidemiológico y clínico en el Hospital Nacional Cayetano Heredia.
}

Tuberculosis in diabetic patients: An epidemiologic and clinical study at the Hospital Nacional Cayetano Heredia.

\author{
DELGADO ROSPIGLIOSI Juan Luis, SECLEN SANTISTEBAN Segundo Nicolás ${ }^{1}$, GOTUZZO HERENCIA, \\ Eduardo ${ }^{2}$.
}

SUMMARY

Objectives: To describe epidemiological and clinical characteristics of diabetic patients with tuberculosis at the Hospital Nacional Cayetano Heredia (HNCH). Material and methods: Retrospective, observational, descriptive study. Diabetes Mellitus (DM) patients with Tuberculosis (TB) were found by searching the register books of the Endocrinology Service, Medicine in-patients Unit and Infectious and Tropical Diseases Department at the HNCH between 1997-2003. Results: There were 85 episodes on TB in 74 analyzed charts. The mean age was $49.4 \pm 12.8$ years; $65 \%$ males, 35\% females. $72 / 74(97.3 \%)$ patients had type 2 DM. Patients were diagnosed with DM a mean of $4.2+4$ years prior to development of TB. $75 / 85(88.2 \%)$ cases presented with pulmonary TB, of whom $51.3 \%$ were diagnosed by positive sputum smear. 59/85 $(69 \%)$ cases were not regularly compliant with the treatment for DM and 60/85 $(71 \%)$ had poor metabolic control when they developed TB. $31 / 74$ (42\%) patients were hospitalized because of TB. 51.4\% of the hospitalizations were associated with diabetic ketoacidosis (DKA). TB can be a leading cause of DKA in our patients. The rate of treatment failures was 15/85 (17.6\%); there was suspicion of MDR-TB in 13/74 (17.6\%) patients. The rate of relapses was 15/74 (20.3\%). 3/74 (4\%) patients died during the period of study. Conclusions: Diabetic patients with poor metabolic control and TB frequently develop relapses, treatment failures and MDR-TB, raising significant concerns about nosocomial transmission. (Rev Med Hered 2006;17:132-140).

KEY WORDS: Tuberculosis, diabetes mellitus.

\section{RESUMEN}

Objetivo: Describir las características epidemiológicas y clínicas de pacientes diabéticos con tuberculosis atendidos en el Hospital Nacional Cayetano Heredia (HNCH). Material y Métodos: Estudio retrospectivo, observacional, descriptivo. Se identificaron los “casos" (pacientes diabéticos con TBC) revisando los registros del Servicio de Endocrinología, Servicios de hospitalización de Medicina y del Departamento de Enfermedades Infecciosas y Tropicales del HNCH atendidos durante el periodo de 1997 al 2003. Resultados: Se encontraron 85 episodios de TBC en 74

\footnotetext{
$1 \quad$ Profesor Principal del Departamento de Medicina, Facultad de Medicina Alberto Hurtado, Universidad Peruana Cayetano Heredia. Director General del Hospital Nacional Cayetano Heredia.

2 Profesor Principal del Departamento de Medicina de la Facultad de Medicina Alberto Hurtado, Universidad Peruana Cayetano Heredia. Departamento de Enfermedades Transmisibles y Dermatológicas. Director del Instituto de Medicina Tropical Alexander von Humboldt Facultad de Medicina Alberto Hurtado, Universidad Peruana Cayetano Heredia
} 
historias clínicas revisadas. El promedio de edad fue de 49,4 £12,8 años; 48/74 (65\%) fueron hombres y 26/74 (35\%) mujeres. 72/74 (97,3\%) pacientes presentaron diabetes mellitus tipo 2. El tiempo promedio de enfermedad diabética previo al desarrollo de TBC fue de $4.2 \pm 4$ años. 75/85 (88,2\%) episodios presentaron TBC pulmonar, habiendo confirmación bacteriológica en el 51,3\% de estos casos. 59/85 (69\%) casos no cumplían regularmente con el tratamiento indicado para la diabetes y 60/85 (71\%) tenían mal control metabólico al desarrollar TBC. 31/74 (42\%) pacientes fueron hospitalizados por TBC, asociándose cetoacidosis diabética (CAD) en el 51,4\% de las hospitalizaciones por esta causa. La TBC puede ser causa importante desencadenante de CAD en nuestro medio. La tasa de fracasos al tratamiento fue de 15/85 (17,6\%); hubo sospecha de TB-MDR en 13/74 pacientes (17,6\%). La tasa de recaídas fue de 15/74 (20,3\%). Fallecieron 3/74 pacientes (4\%) durante el período de estudio. Conclusiones: Pacientes diabéticos mal controlados desarrollan TBC con una elevada tasa de recaídas, fracasos y TB-MDR, con alta sospecha de contagio intrahospitalario. (Rev Med Hered 2006;17:132-140).

PALABRAS CLAVE: Diabetes mellitus, tuberculosis, epidemiología.

\section{INTRODUCCION}

La diabetes mellitus (DM) es una enfermedad metabólica crónica, caracterizada por hiperglicemia y por alteraciones en el metabolismo de los carbohidratos, lípidos y proteínas. La prevalencia en la población peruana varía desde $2 \%$ en la altura hasta $7,6 \%$ en la costa (1). Constituye una de las principales causas de defunción dentro de las enfermedades no transmisibles con una alta tasa de mortalidad en el Perú y el mundo $(1,2,3)$.

Diversos aspectos de la inmunidad se encuentran alterados en los pacientes con DM. La inmunidad celular innata parece ser la más afectada. La función de los leucocitos PMN está deprimida, además la adherencia, la quimiotaxis, la fagocitosis y la destrucción intracelular están disminuidas.

La inmunidad celular adaptativa también se ve afectada en los pacientes diabéticos, con una disminución de la respuesta proliferativa linfocítica a estímulos y a algunos patógenos (4).

Estas alteraciones inmunes determinan que algunas infecciones como la TBC tiendan a ser más comunes o más severas en pacientes diabéticos y que otras ocurran casi exclusivamente en ellos, aumentando el riesgo de complicaciones y mortalidad en estos pacientes $(4,5,6)$.

La TBC continúa siendo uno de los principales problemas mundiales. Dentro de las enfermedades infecciosas, luego del VIH/SIDA, la TBC es la segunda causa mundial de muertes en el mundo, matando cerca de 2 millones de personas cada año y cerca de 300000 pacientes con SIDA. En el año 2002, el Perú permanecía en la lista de los 22 países responsables del $80 \%$ de nuevos casos diagnosticados con TBC en todo el mundo (7).

El Servicio de Neumología y el Departamento de Enfermedades Infecciosas y Tropicales del HNCH son centros importantes de referencia para pacientes con enfermedades infecciosas en nuestro medio, siendo el Programa de Control de la Tuberculosis (PCT) del $\mathrm{HNCH}$ un eje importante en el manejo de pacientes infectados con TBC en Lima Norte.

La TBC puede estar asociada a otras enfermedades; las enfermedades subyacentes más comunes en huéspedes comprometidos con TBC pulmonar son la infección por VIH (7), diabetes mellitus y varios tipos de neoplasia (8). La asociación de TBC en pacientes inmunocomprometidos como en el caso del VIH/SIDA, conlleva un mayor número de complicaciones observándose formas atípicas y más severas de la enfermedad.

Numerosos estudios han publicado la alta prevalencia de la TBC entre diabéticos, con una mayor prevalencia de TBC en diabéticos que en no diabéticos. Las alteraciones inmunes presentes en los pacientes diabéticos, sobretodo en los mal controlados, predisponen a una susceptibilidad mayor y a una forma más severa de TBC (5,8-10).

El objetivo de este estudio fue describir las características epidemiológicas y clínicas de los pacientes diabéticos que desarrollaron tuberculosis atendidos en el $\mathrm{HNCH}$.

\section{MATERIALES Y METODOS}

Se realizó un estudio retrospectivo observacional de tipo descriptivo. Se identificaron los “casos” (pacientes diabéticos con tuberculosis) revisando los registros del 
Servicio de Endocrinología, de los Servicios de hospitalización de Medicina y del Departamento de Enfermedades Infecciosas, Tropicales y Dermatológicas del HNCH, de los pacientes atendidos durante el periodo de 1997 al 2003. Fueron seleccionados para el estudio los casos con DM ya reconocida que desarrollaron TBC en el transcurso de su enfermedad, y que ingresaron a tratamiento regular y supervisado del Programa Nacional de Control de la Tuberculosis (PNCT).

Se programó además la revisión de historias clínicas y tarjetas de control de asistencia y administración de medicamentos del PNCT de los pacientes controlados en el HNCH y de los derivados a Centros de Salud.

La información fue recolectada en fichas especialmente diseñadas para los objetivos del estudio con datos relacionados al curso y control metabólico de la DM, y a la evolución de la TBC.

El control metabólico de la DM fue definido como malo por $\mathrm{HbA}_{1 \mathrm{C}}>7,5 \%$, glicemia en ayunas $>126 \mathrm{mg} / \mathrm{dl}$ y glicemia postprandial $>180 \mathrm{mg} / \mathrm{dl}$; aceptable por $\mathrm{HbA}_{1 \mathrm{C}}$ 7-7,5\%, glicemia en ayunas $110-126 \mathrm{mg} / \mathrm{dl}$ y glicemia postprandial $141-180 \mathrm{mg} / \mathrm{dl}$; y control ideal de la enfermedad por $\mathrm{HbA}_{1 \mathrm{C}}<7 \%$, glicemia en ayunas 80 $109 \mathrm{mg} / \mathrm{dl}$ y glicemia postprandial $80-140 \mathrm{mg} / \mathrm{dl}$.

La multidrogorresistencia (MDR) en TBC se definió por la presencia de resistencia simultánea a isoniacida y rifampicina. Operacionalmente se considera como caso de TB-MDR al paciente con TBC pulmonar bacteriológicamente positiva que:

a) Fracasa al esquema I en condiciones de tratamiento regular y supervisado,

b) Ingresa como recaída o abandono recuperado y fracasa al esquema II en condiciones de tratamiento regular y supervisado.

Una vez que fueron diagnosticados de TBC y entraron a tratamiento regular y supervisado, los pacientes fueron controlados en el hospital o en el centro de salud de referencia donde recibieron tratamiento directamente observado (DOT). En estos lugares se llevó a cabo el control y seguimiento de la enfermedad y se registraron datos como baciloscopías, cultivos, cumplimiento del tratamiento y diagnósticos de alta del PNCT. No se nos permitió el acceso a las tarjetas de control de los pacientes del PCT del HNCH por lo que este estudio se limita a los datos recogidos de las historias clínicas.

Los datos obtenidos de la revisión de historias cínicas fueron ingresados a una base de datos utilizando el software Microsoft Excel XP. Se realizó el análisis de las variables utilizando tablas de frecuencia para las variables nominales y medidas de tendencia central (promedios) para las variables numéricas. Se usó medianas cuando la desviación estándar fue mayor al promedio.

\section{RESULTADOS}

Se lograron identificar 100 casos de los cuales sólo 78 tenían historias clínicas disponibles. En este estudio se consideró cada episodio de TBC (casos nuevos, recaídas) como uno nuevo, obteniéndose 94 episodios; 9 fueron excluidos por no tener diagnóstico de DM al desarrollar TBC, quedando finalmente 85 episodios de TBC para el análisis en 74 pacientes diabéticos conocidos; 15 fueron recaídas y 70 casos nuevos.

Cuarentaidos pacientes (56,8\%) fueron captados en la consulta externa, 27 (36,5\%) en hospitalización y 5 (6,7\%) en Emergencia.

El 97,3\% (72/74) de la población estudiada presentó DM tipo 2 y el 2,7\% (2/74) DM tipo 1. La edad promedio para desarrollar TBC fue 49,4 $\pm 12,8$ años con una edad máxima de 81 y una mínima de 18. El $65 \%$ (48/74) fueron hombres y el 35\% (26/74) mujeres. Los lugares de procedencia más comunes fueron San Martín de Porras (19.5\%), San Juan de Lurigancho (16,9\%), Lima (15,6\%) y los Olivos (12,3\%).

Diabetes mellitus: Tiempo de enfermedad: En promedio la duración de la DM en estos pacientes fue de 5,8 \pm 4,3 años. El tiempo promedio de enfermedad diabética previo al desarrollo de TBC fue de 4,2 \pm 4 años, con un máximo de 19 años y un mínimo de 2 meses.

Complicaciones: Se encontraron evidencias clínicas de complicaciones por DM en 29 pacientes $(39,2 \%)$.

Las complicaciones más frecuentes fueron neuropatía periférica (19\%), nefropatía $(16,2 \%)$ y retinopatía (16,2\%). El 6,8\% presentaba pie diabético, el 5,4\% cardiopatías, el $4 \%$ enfermedad vascular periférica y el $1,4 \%$ antecedente de enfermedad cerebro vascular.

Control metabólico: El 71\% (60/85) presentaba un mal control metabólico al desarrollar TBC, el 8\% (7/ 85) aceptable y sólo el 1\% (1/85) un control ideal de la enfermedad. En el 20\% de los casos (17/85) no se encontraron controles de la DM (Tabla $\mathrm{N}^{\circ} 1$ ).

Tratamiento: Se encontró que sólo el 14\% (12/85) de los pacientes cumplían regularmente con el 
Tabla $N^{\circ} 1$. Control Metabólico de la diabetes mellitus en el momento del diagnóstico de TBC; Hospital Nacional Cayetano Heredia 1997-2003.

\begin{tabular}{ccc}
\hline & Total & \% \\
\hline Ideal* & 1 & 1 \\
Aceptable** & 7 & 8 \\
Malo*** & 60 & 71 \\
Sin Control & 17 & 20 \\
Total General & 85 & 100 \\
\hline
\end{tabular}

* Ideal: $\mathrm{HbA}_{1 \mathrm{C}}<7 \%$, glicemia en ayunas $80-109 \mathrm{mg} / \mathrm{dl}$ y glicemia postprandial $80-140 \mathrm{mg} / \mathrm{dl}$.

** Aceptable: $\mathrm{HbA}_{1 \mathrm{C}}$ 7-7.5\%, glicemia en ayunas 110$126 \mathrm{mg} / \mathrm{dl}$ y glicemia postprandial 141-180mg/dl.

*** Malo: $\mathrm{HbA}_{1 \mathrm{C}}>7.5 \%$, glicemia en ayunas $>126 \mathrm{mg} / \mathrm{dl} \mathrm{y}$ licemia postprandial $>180 \mathrm{mg} / \mathrm{dl}$.

tratamiento antidiabético indicado. La mayor parte (69\%; 59/85) lo hacía de forma irregular y el 1,2\% (1/ 85) no recibía ningún tipo de tratamiento para la diabetes. El dato no se precisaba en 12/85 episodios (14\%).

Los esquemas de tratamiento encontrados fueron variados y generalmente dependieron del grado de control metabólico que presentaban los pacientes en las consultas. El 34\% de los casos (29/85) fueron manejados con insulina y antidiabéticos orales (ADO) en algún momento de la enfermedad. El 23,5\% (20/ 85) fueron manejados sólo con ADO y el 14\% (12/85)

Tabla N³. Diagnósticos de alta del PCT y evolución de los pacientes diabéticos con TBC del HNCH 1997-2003.

\begin{tabular}{|c|c|c|c|}
\hline & $\begin{array}{c}\text { TBC } \\
\text { primaria } \\
(n=70)\end{array}$ & $\begin{array}{c}\text { TBC } \\
\text { secundaria } \\
(n=15)\end{array}$ & $\begin{array}{c}\text { Total } \\
\text { General } \\
(n=85)\end{array}$ \\
\hline Curados & 17 & 1 & 18 \\
\hline Abandonos & 3 & - & 3 \\
\hline Fracasos & 8 & 8 & 16 \\
\hline TB-MDR & 6 & 7 & 13 \\
\hline Fallecidos & 3 & - & 3 \\
\hline
\end{tabular}

Tabla $N^{\circ} 2$. Tipo de tratamiento para la diabetes mellitus en pacientes con TBC; Hospital Nacional Cayetano Heredia 1997-2003.

\begin{tabular}{lcc}
\hline & Total & \% \\
\hline Antidiabéticos orales + Insulina & 29 & 34 \\
Antidiabéticos orales & 20 & 23,5 \\
Insulina & 12 & 14,1 \\
Antidiabéticos orales + Insulina + Dieta & 8 & 9,4 \\
Antidiabéticos orales + Dieta & 7 & 8.2 \\
Insulina + Dieta & 5 & 6 \\
No especificado & 2 & 2,4 \\
Dieta & 1 & 1,2 \\
Sin tratamiento & 1 & 1,2 \\
Total General & 85 & 100 \\
\hline
\end{tabular}

sólo con insulina. El uso de dieta en el tratamiento de estos pacientes generalmente se encontraba asociado a ADO (8.2\%), a insulina (6\%), y a ADO e insulina $(9,4 \%)$. La dieta como tratamiento único, fue encontrada en el 1,2\% de los casos (1/85) (Tabla N²).

Episodio de tuberculosis: Se encontró el seguimiento de 34 episodios hasta terminar el tratamiento anti-TBC (18 curados y 16 fracasos al tratamiento) y de 3 que lo abandonaron. En los 48 episodios restantes no se encontró el seguimiento completo de los pacientes hasta finalizar el tratamiento. En la tabla $N^{\circ} 3$ se señalan los

Tabla No 4. Causas de hospitalización en pacientes diabéticos con TBC en el Hospital Nacional Cayetano Heredia 1997-2003.

\begin{tabular}{lcc}
\hline & Total & $\%$ \\
\hline TBC & 35 & 43 \\
Cetoacidosis diabética & 24 & 30 \\
Pie diabético & 6 & 7,4 \\
No especificado & 5 & 6,2 \\
Hemorragia digestiva alta & 3 & 3,7 \\
Celulitis & 2 & 2,5 \\
Hipoglicemia & 2 & 2,5 \\
Colecistitis crónica calculosa & 2 & 2,5 \\
Otras & 2 & 2,2 \\
Total General & 81 & 100 \\
\hline
\end{tabular}


diagnósticos de alta del PCT y la evolución de los pacientes.

Forma clínica: El 88,2\% (75/85) de los pacientes desarrollaron la forma pulmonar y el 9,4\% (8/85) formas extrapulmonares y mixtas. Tres pacientes $(3,5 \%)$ presentaron TBC pleural, 4 (4,7\%) pleuroparenquimal y uno $(1,2 \%)$ meningoencefalitis-TB y pulmonar.

Diagnóstico: La confirmación bacteriológica (baciloscopía) se encontró en el 51,3\% (41/80) de los casos pulmonares. La baciloscopía fue de $1+$ en el $39 \%$ de casos (31/80), 2+ en el 7,5\% (6/80) y $3+$ en el $5 \%$ $(4 / 80)$. No se especificaba el dato en el 33\% (26/80) de los casos.

Reacción adversa a fármacos antituberculosos (RAFA): Las RAFA estuvieron presentes en 8 pacientes $(10,8 \%)$. La mediana de meses para desarrollar RAFA fue de 2 con un máximo de 18 y un mínimo de 1 mes. En 5 pacientes se indicó un nuevo tratamiento, en uno se suspendió y en los 2 restantes no se especificaba el manejo de RAFA.

Hospitalizaciones: El 42\% (31/74) de los pacientes fueron hospitalizados por lo menos una vez por alguna complicación de la TBC y el 5,4\% (4/74) dos veces. TBC fue la principal causa de hospitalización (43, seguida de cetoacidosis diabética (CAD) (30\%) y pie diabético $(7,4 \%)$, entre otras causas (Tabla $\mathrm{N}^{\circ} 4$ ). Síndrome de dificultad respiratoria fue el motivo de hospitalización por TBC más frecuente encontrándose en 15 casos (43\%), seguido de hemoptisis en 9 casos $(25,8 \%)$. CAD se encontró asociada además a más de la mitad de las hospitalizaciones por TBC $(51,4 \%$; 18 / $35)$, y a otras causas menos frecuentes como pie diabético (3/6), celulitis $(1 / 2)$ y hemorragia digestiva alta (HDA) (1/3).

Recaídas: La mediana de años de observación fue de 1, con un máximo de 16 años y un mínimo de 2 meses de observación por episodio. En este periodo de tiempo la tasa de recaídas fue de 20,3\% (15/74).

Fracasos y Resistencia a drogas: La tasa de fracasos al tratamiento antituberculoso por episodio de TBC fue de $18,8 \%$ (16/85), $11,4 \%$ (8/70) en primarios y 53,3\% (8/15)en secundarios. Recibieron esquema de tratamiento estandarizado para TB-MDR 17,6\% (13/ $74), 8,6 \%(6 / 70)$ en primarios y $47 \%(7 / 15)$ en secundarios (Tabla $\mathrm{N}^{\circ} 5$ ).

Fallecimiento: $3 / 74$ pacientes (4\%) fallecieron durante el período de observación de este estudio.
Tabla N5. Tasa de TBC-MDR en pacientes diabéticos del Hospital Nacional Cayetano Heredia 1997-2003.

\begin{tabular}{ccc}
\hline & $\begin{array}{c}\text { Total MDR } \\
(\mathbf{n = 1 3})\end{array}$ & $\mathbf{\%}$ \\
\hline TBC primaria & $6 / 70$ & $8,6 \%$ \\
TBC secundaria & $7 / 15$ & $46,7 \%$
\end{tabular}

\section{DISCUSION}

Los resultados obtenidos en este estudio muestran una población de pacientes diabéticos adultos mayores con pobre control metabólico en su mayoría que desarrollan TBC con una tasa alta de recaídas y fracasos al tratamiento, asimismo multidrogorresistencia elevada.

La DM es una enfermedad crónica en la que la duración de la enfermedad y el mal control metabólico influyen directamente en la aparición de complicaciones. Un $20-30 \%$ de los pacientes con DM que conocen su enfermedad no cumplen ningún tipo de tratamiento y el $68 \%$ de los casos se diagnostican de forma casual, generalmente a consecuencia de la manifestación clínica de alguna de sus complicaciones crónicas.

Debido a esto, alrededor de dos tercios de la población con DM tiene o está en riesgo de sufrir complicaciones crónicas (11), siendo estos, factores que favorecen el desarrollo de infecciones (12). En esta serie se encontraron evidencias clínicas de complicaciones de la DM en el 39,2\% de pacientes, siendo neuropatía periférica, nefropatía y retinopatía las más frecuentes. Yamagishi y col observaron que la frecuencia de complicaciones de la DM en pacientes con TBC es alta. En hombres la frecuencia de complicaciones era el doble que en mujeres mostrando un pico entre los 40 y 50 años en hombres y 60 años en mujeres (13).

En un estudio en India se comparó además la duración de la diabetes mellitus en pacientes DM/TBC resistentes y no resistentes a drogas antituberculosas, encontrándose una mayor duración de la enfermedad en los pacientes DM/TBC resistentes (5,8 y de 3,7 años de enfermedad respectivamente (14). En este estudio encontramos comparativamente una duración de 7,1 y de 5,6 años de la diabetes en pacientes MDR y noMDR respectivamente.

El buen control metabólico de la DM, que implica mantener glicemias en límites normales, hemoglobina glicosilada $\left(\mathrm{HbA}_{1 \mathrm{C}}\right)$ menor de 7,0\% y perfil lipídico sin riesgo cardiovascular, es difícil de lograr a pesar de los 
avances en el tratamiento, siendo muy frecuente encontrar pacientes diabéticos con mal control de la enfermedad quienes se comportan como inmunosuprimidos (15).

La hiperglicemia y los productos finales de la glicosilación avanzada llevan a un estado persistente de pobre nivel de activación de los leucocitos PMN. Hay una significativa correlación negativa entre los niveles de $\mathrm{HbA}_{1 \mathrm{C}}$ y la actividad bactericida de los neutrófilos. Por otra parte, el sistema antioxidante involucrado en la actividad bactericida se ve alterado, encontrándose que el buen control de la glicemia rectifica algunas de estas deficiencias $(5,16)$.

En América Latina, según los indicadores de educación terapéutica, sólo la mitad o la cuarta parte de los pacientes diabéticos podrían desempeñar un papel activo y eficaz en el control y tratamiento de su enfermedad (11). En este estudio se encontró una elevada tendencia al incumplimiento y al mal control de la DM; el 71\% tenía mal control metabólico, el 20\% no se controlaba la enfermedad y casi el $70 \%$ no cumplía regularmente con el tratamiento indicado. Esto se debe principalmente a que los pacientes no adquieren la educación necesaria para colaborar con el control de la diabetes (12) y a los escasos recursos económicos que dificultan el acceso a dietas y medicamentos en nuestro país, lo cual predispone al desarrollo de complicaciones.

En los pacientes diabéticos con TBC se recomienda el uso de insulina para el tratamiento e incluso se contraindica ciertos antidiabéticos orales (ADO). Estudios encuentran un efecto protector de la insulina en estos pacientes (17). Sin embargo los resultados obtenidos en este estudio muestran que $75 \%$ fueron manejados con ADO y 64\% con insulina durante el tratamiento. El 34\% de nuestros pacientes usó ADO e insulina durante el tratamiento y sólo el 14\% únicamente insulina.

Las principales armas con las que cuenta el huésped en la defensa del organismo contra el BK son los macrófagos activados, que pueden matar o inhibir al BK que ingieren (inmunidad mediada por células, resistencia celular adquirida) y la habilidad de frenar el crecimiento intracelular del bacilo dentro de un macrófago no activado formando tejido caseoso sólido (hipersensibilidad retardada) (18). Las alteraciones inmunes presentes en los pacientes diabéticos mal controlados afectarían principalmente estas respuestas normales del organismo (adherencia, quimiotaxis, fagocitosis, destrucción intracelular), generando pacientes más susceptibles y formas más severas de la TBC.

Estudios en Japón encuentran a la DM como la enfermedad subyacente más común en pacientes con TBC pulmonar activa, y una mayor proporción de muestras de esputo positivas en pacientes diabéticos con $\mathrm{HbA}_{1 \mathrm{C}}$ elevada (19). Asimismo, en el 2004 Yshii y col encontraron en el HNCH y Centros de Salud de Lima Norte presentaciones radiológicas atípicas y una peor evolución clínica y bacteriológica en pacientes con TBC y diabetes mellitus (17).

En el seguimiento de nuestros pacientes (diabéticos conocidos) se observó también las hospitalizaciones que presentaron. Se encontró que la causa más común de hospitalización fue TBC seguida de cetoacidosis diabética (CAD).

Las infecciones son el principal factor precipitante de CAD (20). En nuestro país por ejemplo, están presentes en el 67\% de casos atendidos por CAD en el $\mathrm{HNCH}$ y en el 55\% de los atendidos en el Hospital Nacional Dos de Mayo (HNDM) $(21,22)$. En esta serie de casos, CAD se asoció a infecciones como pie diabético, celulitis, y al 51.4\% de las hospitalizaciones por TBC, estando presente en el 58\% del total de hospitalizaciones ya sea como factor causal o asociado.

Se estima que la TBC pulmonar es de 3 a 4 veces más frecuente en diabéticos que en la población general (9). La frecuencia con la que los pacientes diabéticos acuden a hospitales y centros de salud para hospitalización, emergencias y/o consulta ambulatoria es alta, aumentando el riesgo de contagio de TBC intranosocomial.

Previo a la epidemia del VIH, aproximadamente el $84,6 \%$ de los casos reportados de TBC estaban limitados al pulmón y un 14,6\% involucrando formas extrapulmonares y/o mixtas (23). En el Perú, encontramos que la incidencia de tuberculosis pulmonar para el 2000 fue de 111,4 por 100000 habitantes y de extrapulmonar de 22,1 por 100000 habitantes (24). En este estudio el 88,2\% desarrolló TBC pulmonar y el $9,4 \%$ formas extrapulmonares y mixtas. Más de la mitad de los casos pulmonares fueron $\mathrm{BK}(+)$, comparable a lo encontrado en otras series $(24,25)$. Esto es similar a la población no diabética con TBC pero diferente a la población TBC/VIH (7).

DM es una de las patologías asociadas a RAFA más frecuentes, con un 5-7\% de asociación entre los años 1991-2000 (26). En este estudio se encontró que 10,8\% presentó RAFA, condición que representa un riesgo de irregularidad y abandono al tratamiento. 
La reactivación de la tuberculosis latente es presumiblemente el resultado de una falla en la inmunidad, como se describe en la infección por VIH $(25,27)$. En la serie de pacientes diabéticos de Arbañil y col en el HNDM, se encontró una reactivación de la TBC en el 21\% de casos (28). Las recaídas se producen ya sea por reactivación de la TBC latente o por reinfección exógena con una nueva cepa, como se demuestra en diversos estudios moleculares $(29,30)$.

El índice de casos de TBC notificados al ingreso como recaídas en el Perú para el 2000 fue de 11\%. La tasa anual de recaídas por primera vez fue de 17,1 por 100000 habitantes y de 2,4 por 100000 habitantes por segunda o más veces (24). En este estudio 70 episodios fueron TBC primaria y 15 TBC secundaria, mostrando una tasa de recaídas de 20,3\%.

Otros estudios sugieren además una alta tasa de falla en la respuesta al tratamiento de la TBC pulmonar en $\mathrm{DM}$, con un aumento en la frecuencia de cepas resistentes a la farmacoterapia (10,31). En este estudio encontramos que la tasa de fracasos al esquema I y II fue de $11,4 \%$ y $53,3 \%$ respectivamente, cifras bastante elevadas comparándolas con la población general de pacientes con TBC en el Perú, en quienes se logró obtener para el año 2000 92,9\% de eficiencia al esquema I y una tasa de fracasos al esquema I y II menor al 5\% (32). Voloshyn y col (33) encontraron en Ucrania que los principales factores asociados a quimioterapia inefectiva en pacientes con TBC pulmonar y DM fueron la severidad del curso de la DM y sus complicaciones, la poca adherencia de los pacientes al tratamiento, la diseminación de la tuberculosis y la resistencia del bacilo tuberculoso.

En los últimos años, se han reportado numerosos brotes de TB-MDR en el mundo principalmente en pacientes infectados con VIH/SIDA, quienes se piensa han sido expuestos a estas cepas en centros médicos o correccionales $(30,34,35)$. La multidrogorresistencia afecta operacionalmente a los pacientes que fracasan al esquema I y al esquema II en condiciones de tratamiento regular y supervisado. La tasa de pacientes que iniciaron esquema de tratamiento estandarizado para TB-MDR encontrada en este estudio fue de 17,6\% (13/ 74). De los 13 pacientes con TB-MDR, 6 eran TBC primaria, mostrando una tasa cercana al 9\% (6/70), cifra más elevada que el $3 \%$ de MDR primaria que se espera tener en el Perú (36). Kameda y col encontraron en Japón que los pacientes diabéticos recaen con cepas resistentes y tienen un pronóstico grave una vez dada la recaída (37).
De las 15 recaídas encontradas en este estudio el 47\% fueron TB-MDR, siendo esta cifra muy elevada comparándola con el $12.3 \%$ de MDR adquirida que se tiene en el Perú (36). Tres pacientes que fracasaron al tratamiento anti-TBC (Dos primarios y uno secundario) no han sido considerados como MDR en este estudio, debido a que no se encontraron evidencias en las historias clínicas de que hayan iniciado el esquema de tratamiento estandarizado para TB-MDR, por lo que podríamos estar subestimando la cifra de MDR en esta población. La posibilidad de contagio intranosocomial de la TB-MDR en estos pacientes podría ser alta, como se describe en pacientes inmunocomprometidos con VIH/SIDA, neoplasias, terapia inmunosupresora, etc, e inmunocompetentes como trabajadores de salud $(30,34,38-40)$.

Si bien la pérdida inicial del 22\% de la muestra debilita la fortaleza de nuestros resultados, pese a ser una población pequeña, el número de casos que recaen con cepas MDR es muy elevado por lo que debe tomarse en consideración. Una de las limitaciones de este estudio es ser retrospectivo, pudiendo haberse perdido información valiosa. Otra limitación importante se presentó durante la recolección de la información. A pesar de las reiteradas peticiones, en el PCT del HNCH no se nos permitió el acceso para la revisión de tarjetas de control y administración de medicamentos de los pacientes diabéticos por no haber sido un estudio originado en su servicio, lo que generó un sesgo de información. Esto llevó además a tener la documentación del diagnóstico de TBC en sólo el 51\% de los episodios, quedando duda de la certeza diagnóstica en casi la mitad de la población, a pesar de haber iniciado tratamiento regular y supervisado. A pesar de esto y con el seguimiento hecho a los pacientes durante sus controles en las historias clínicas, los datos obtenidos en este estudio no dejan de tener un importante valor referencial para iniciar estudios posteriores en esta población. Además, la falta de información en el seguimiento hasta el término del tratamiento anti-TBC en un gran número de episodios (48/85), podría señalar que la morbilidad y mortalidad en esta población fueran aún más altas; lo que se muestra es el escenario mínimo.

Según lo observado en este estudio, la posibilidad de transmisión de TB-MDR intrahospitalaria en pacientes diabéticos mal controlados con alteraciones en la respuesta inmunitaria normal que acuden en gran número a controles, emergencias y salas de hospitalización podría ser alta. Factores como la duración de la diabetes, el tratamiento antidiabético 
usado y el cumplimiento del mismo, asi como las RAFA, podrian influir en aumentar el riesgo de contagio. Existe la necesidad de comprender los medios por los cuales se desarrolla esta enfermedad, para poder idear e implementar medidas de seguridad y prevención adecuadas.

En conclusión, los resultados obtenidos en este estudio presentan a una población diabética predominantemente masculina, adulta mayor y con un deficiente control metabólico al desarrollar TBC. La TBC puede ser causa importante desencadenante de cetoacidosis diabética en nuestro medio. A pesar de ser una población pequeña, el porcentaje de recaídas y fracasos al tratamiento antituberculoso fue alto, así como una elevada tasa de TB-MDR, al comparar estos datos con los obtenidos en otros estudios.

Esperamos que este estudio sea un punto de partida que invite a seguir investigando en estas dos enfermedades que cada día se vuelven más prevalentes, para mejorar la calidad de vida de los pacientes, optimizar el uso de recursos e implementar medidas de seguridad y preventivas en la transmisión de TBC en pacientes inmunocomprometidos.

\section{Agradecimiento:}

Al doctor Hugo Arbañil del Servicio de Endocrinología del Hospital Dos de Mayo por su apoyo en la obtención de información, al Dr.Arturo Villena por su tiempo y dedicación, y un agradecimiento muy especial a la Doctora Elsa Gonzales Lagos que sin su ayuda no hubiera sido posible la realización de este trabajo.

\section{Correspondencia:}

Juan Luis Delgado Rospigliosi

Malecón de la Marina 1080 Dpto. 503, Miraflores.

Lima, Perú.

Teléfonos: 511-9851 4218 / 511-224 1040

Correo electrónico: derpapu@yahoo.com

\section{REFERENCIAS BIBLIOGRAFICAS}

1. Seclén S. La Diabetes mellitus - Problema de salud pública en el Perú. Lima-Perú: News Graf SA; 2000.

2. World Health Organization. WHO Study Group. World Health Organization Technical report series Nro 727. Genova:WHO; 1985.

3. Barceló A, Rajpathak S. Incidence and prevalence of diabetes mellitus in the Americas. Rev Panam Salud Publica 2001; 10(5): 300-308.

4. Calvet H, Yoshikawa T. Infections in diabetes. Infectious Disease Clinics of North America 2001;15(2):25-32.
5. Nirmal J, Caputo G, Wietekamp M, et al. Primary care: Infections in patients with diabetes mellitus. NEJM 1999; 341(25):1906-12.

6. Koziel H, James M. Pulmonary complications of diabetes mellitus: Pneumonia. Infectious Disease Clinics of North America 1995; 9(1):31-44.

7. Frieden T, Sterling T, Munsiff S, et al. Seminar: Tuberculosis. Lancet 2003; 362: 887-899.

8. Ezung T, Devi NT, Singh NT, Singh TB. Pulmonary tuberculosis and diabetes mellitus-a study. J Indian Med Assoc 2002; 100(6): 376, 378-9.

9. Kim SJ, Hong YP, Lew WJ, Yang SC, Lee EG. Incidence of pulmonary tuberculosis among diabetics. Tuberc Lung Dis 1995 ; 76(6): 529-33.

10. Mboussa J, Monabeka H, Kombo M, et al. Course of pulmonary tuberculosis in diabetics. Rev Pneumol Clin 2003; 59(1): 39-44.

11. Gagliardino JJ, De la Hera M, Siri F, et al. Evaluación de la calidad de la asistencia al paciente diabético en América Latina. Rev Panam Salud Pública 2001; 10 (5): 309-317.

12. Korhonen T, Huttunen JK, Aro A, et al. A controlled trial on the effects of patients education in the treatment of insulin-dependent diabetes. Diabetes Care 1983; 6:256-61.

13. Yamagishi F, Sasaki Y, Yagi T, et al. Frequency of complication of diabetes mellitus in pulmonary tuberculosis. Kekkaku 2000; 75(6): 435.

14. Subhash HS, Ashwin I. Drug resistant tuberculosis in diabetes mellitus: A retrospective study from South India. Trop Doct 2003; 33 (3): 154.

15. Kolterman OG, Olefsky JM, Jurahara C, Taylor K. A defect in cell-mediated inmune function in insulinresistant diabetic and obese subjects. J Lab Clin Med 1980; 956:535-43.

16. Arnstein R.. Endocrine and Metabolic aspects of tuberculosis. En: Schlossberg D. Tuberculosis. New York: Springer-Verlag Inc; 1994. p. 247-256.

17. Yshii CH, Accinelli RA, Alvarez LM, et al. Clinical presentation, radiological findings and prognosis of tuberculosis patients with diabetes mellitus: A casecontrol study. AJRCCM 2004; 169(7): A134.

18. Dannenberg AM Jr. Pathogenesis and immunology: Basic aspects. En: Schlossberg D. Tuberculosis. New York: Springer-Verlag Inc 1994. p. 17-39.

19. Tamura M, Shirayama R, Kasahara R, et al. A study on relation between active pulmonary tuberculosis and underlying diseases. Kekkaku 2001; 76(9): 619.

20. American Diabetes Association. Hyperglycemic crises in patients with Diabetes Mellitus. Diabetes Care 2003; 26(1): S109-117.

21. Villena J, Villena A, Seclén S, et al. Evaluación clínica y manejo de la Cetoacidosis Diabética. Lima- Perú: I Congreso Peruano de Endocrinología. Resumen 25. Nov. 21-25, 1985.

22. Arbañil H, Valdivia H, Zubiate C, Murguia C, Paz C. Aspectos clínicos y terapéuticos de la Cetoacidosis diabética en el Hospital Dos de Mayo 1992. Lima, Perú: I Congreso Nacional de Diabetes Mellitus: Resumen 5;1992. 
23. Hopewell PC. Overview of clinical Tuberculosis. En: Bloom B. Tuberculosis: Pathogenesis, protection and control. Washington: American Society for Microbiology, 1994. p. 25-46.

24. Dirección General de Salud de las Personas / Programa Nacional de Control de Enfermedades Transmisibles / Programa Nacional de Control de la Tuberculosis Ministerio de Salud del Peru. Tuberculosis en el Perú: Informe 2000. Lima: Tarea Asociación Gráfica Educativa; 2001. p. 302.

25. Smith PG, Moss AR. Epidemiology of tuberculosis. En: Bloom B. Tuberculosis: pathogenesis, protection and control. Washington: American Society for Microbiology; 1994. p. 47-59.

26. Bonilla C, Suárez PG, Portocarrero J, et al. Patrón de presentación de reacciones adversas a fármacos antituberculosos en tratamientos directamente observados, Perú 1991-2000. En: Dirección General de Salud de las Personas / Programa Nacional de Control de Enfermedades Transmisibles / Programa Nacional de Control de la Tuberculosis - Ministerio de Salud del Perú. Tuberculosis en el Perú: Informe 2000. Lima: Tarea Asociación Gráfica Educativa; 2001. p. 107-120.

27. Haas D. Mycobacterium tuberculosis. En: Mandell G, Bennett J, Dolin R Principles and Practice of Infectious Diseases,. Philadelphia, Pennsylvania: Churchill Livingstone, 2000. p. 2576-2580.

28. Arbañil HC. Infecciones en el diabético. En: Calderon R, Peñaloza JB. Diabetes mellitus en el Perú. Lima: Desa;1996. p . 201-211.

29. Van Rie A, Warren R, Richardson M, et al. Exogenous reinfection as a cause of recurrent tuberculosis after curative treatment. NEJM 1999; 341(16): 1174-9.

30. Small PM, Shafer RW, Hopewell PC, et al. Exogenous reinfection with multidrug-resistant Mycobacterium tuberculosis in patients with advanced HIV infection. NEJM 1993; 328(16): 1137-44.

31. Voloshyn IM, Kovalechenko VF. Efficiency of surgical treatment of patiens with pulmonary tuberculosis and diabetes mellitus in conditions of the mycobacterial tuberculosis resistant to pharmacotherapy. Klin Khir
2000; (7): 38.

32. Zavala D. Evaluación por estudio de cohorte del tratamiento antituberculoso diferenciado en el Perú, años 1991-2000. En: Dirección General de Salud de las Personas / Programa Nacional de Control de Enfermedades Transmisibles / Programa Nacional de Control de la Tuberculosis - Ministerio de Salud. Tuberculosis en el Perú: Informe 2000. Lima: Tarea Asociación Gráfica Educativa; 2001. p. 41-49.

33. Voloshyn IM, Kovalechenko VF, Podhaievs'kyi SG. Causes of inefective chemotherapy of pulmonary tuberculosis with diabetes mellitus. Klin Khir 1999; (8): 20.

34. Beck-Sague C, Dooley SW, Hutton MD, et al. Hospital outbreak of multidrug-resistant Mycobacterium tuberculosis infections. Factors in transmission to staff and HIV-infected patients. JAMA 1992; 268(10):1280.

35. Flament-Saillour M, Robert J, Jarlier V, et al. Outcome of multidrug-resistant tuberculosis in France. A Nationwide case-control study. Am J Respir Crit Care Med 1999; 160 (2): 587-593.

36. Suárez PG. El control de la tuberculosis en el Perú y sus logros en el periodo 1991-2000. En: Dirección General de Salud de las Personas / Programa Nacional de Control de Enfermedades Transmisibles / Programa Nacional de Control de la Tuberculosis - Ministerio de Salud. Tuberculosis en el Perú: Informe 2000. Lima: Tarea Asociación Gráfica Educativa; 2001. p. 17-24.

37. Kameda K, Kawabata S, Masuda N. Follow-up study of short course chemotherapy for pulmonary tuberculosis complicated with diabetes mellitus. Kekkaku 1990; 65(12): 791.

38. Kenyon T, Ridzon R, Luskin-Hawk R, et al. A nosocomial outbreak of multidrug-resistant tuberculosis. Ann Intern Med 1997; 127(1): 32-36.

39. Schwartzman K, Menzies D. Tuberculosis: 11. Nosocomial disease. CMAJ 1999; 161(10): 1271-7.

40. Narayanan S, Das S, Garg R, et al. Molecular epidemiology of tuberculosis in a rural area of high prevalence in Souh India: Implications of Disease Control and Prevention. J Clin Microbiol 2002; 40(12): 4785-88.

Recibido: 28/03/05

Aceptado para publicación: 10/08/06 